doi: $10.2306 /$ scienceasia1513-1874.2013.39.026

\title{
Characterization of xylose-utilizing yeasts isolated from herbivore faeces in Thailand
}

\author{
Wanlapa Lorliam $^{\mathrm{a}}$, Ancharida Akaracharanya ${ }^{\mathrm{b}}$, Sasitorn Jindamorakot ${ }^{\mathrm{c}}$, Surisa Suwannarangsee ${ }^{\mathrm{d}}$, \\ Somboon Tanasupawat ${ }^{\mathrm{a}, *}$ \\ a Department of Biochemistry and Microbiology, Faculty of Pharmaceutical Sciences, \\ Chulalongkorn University, Bangkok 10330 Thailand \\ b Department of Microbiology, Faculty of Science, Chulalongkorn University, Bangkok 10330 Thailand \\ c BIOTEC Culture Collection, Bioresources Technology Unit, \\ National Centre for Genetic Engineering and Biotechnology (BIOTEC), Pathum Thani 12120 Thailand \\ d Enzyme Technology Laboratory, Bioresources Technology Unit, \\ National Centre for Genetic Engineering and Biotechnology (BIOTEC), Thailand Science Park, \\ Pathum Thani 12120 Thailand
}

${ }^{*}$ Corresponding author, e-mail: Somboon.T@chula.ac.th

Received 18 May 2012

Accepted 26 Nov 2012

\begin{abstract}
A total of 39 xylose-utilizing yeast strains were isolated from herbivore faeces in Thailand. They were identified as Candida tropicalis (32 isolates), Candida albicans (1 isolate), Pichia terricola (1 isolate), Trichosporon mycotoxinivorans (2 isolates), Sporopachydermia lactativora (2 isolates) and Zygoascus meyerae (1 isolate) based on their morphological, cultural, physiological and biochemical characteristics including the sequence analysis of the D1/D2 region of the large-subunit ribosomal DNA. Thirty seven isolates could ferment xylose to ethanol. Zygoascus meyerae E23 isolated from elephant faeces produced the highest ethanol concentration (3.61 g/l after $72 \mathrm{~h})$. C. tropicalis A26 isolated from cow faeces produced the highest xylitol concentration $(43.79 \mathrm{~g} / \mathrm{l})$ which corresponded to $0.71 \mathrm{~g}$ xylitol/g xylose after $24 \mathrm{~h}$. C. tropicalis A26 xylose reductase showed $98.4 \%$ identity and 99.0\% similarity to C. tropicalis (ABX60132C) xylose reductase, and showed the tetra-amino acid motif (Ile-Pro-Lys-Ser) which is conserved among NADPH-dependent xylose reductase.
\end{abstract}

KEYWORDS: Candida, Pichia, Sporopachydermia, Trichosporon, xylose fermentation, xylose reductase gene, Zygoascus

\section{INTRODUCTION}

Lignocellulose is an interesting non-food fermentable sugar resource for ethanol fuel production ${ }^{1}$. It consists of $30-40 \%(\mathrm{w} / \mathrm{w})$ cellulose and $15-25 \%(\mathrm{w} / \mathrm{w})$ hemicellulose $\mathrm{e}^{1,2}$ and it is the world most abundant biomass. Completely hydrolysis of the cellulose and the hemicellulose results in glucose and xylose, respectively, which several microorganisms can ferment to ethanol ${ }^{3}$. Saccharomyces cerevisiae is the most popular yeast for fermenting glucose to ethanol due to its high ethanol production yield and comparatively high ethanol tolerance ${ }^{4,5}$, however $S$. cerevisiae strains cannot ferment xylose to ethanol. Several yeasts such as Candida shehatae, Pachysolen tannophilus, Brettanomyces naardenensis, C. tenuis, Pichia segobiensis, C. lyxosophila, C. intermedia, C. jeffriesii, Spathaspora passalidarum, Spathaspora arborariae, C. prachuapensis, and Scheffersomyces stipitis have been reported as xylose fermenting yeasts ${ }^{6-10}$. The $S$. stipitis strain produces significant amounts of ethanol from xylose and it has been studied extensively ${ }^{9}$.

To increase an ethanol production yield from lignocellulose, both glucose and xylose liberated should be fermented to ethanol. Although S. stipitis strain can ferment both glucose and xylose to ethanol, its ethanol yield from glucose and ethanol tolerance are much lower than those of $S$. cerevisiae ${ }^{9,11}$. Cocultivating of $S$. cerevisiae and $S$. stipitis strains to co-ferment glucose and xylose remains unsatisfactory, due to their difference in fermenting condition and ethanol tolerance ${ }^{12,13}$. S. stipitis strain prefers to ferment glucose more than xylose while it has lower ethanol tolerance than $S$. cerevisiae strain ${ }^{14}$. So xylose was not fermented. Therefore, there has been an 
attempt to construct recombinants $S$. cerevisiae strain capable of fermenting xylose by overexpressing the genes encoding enzymes in the xylose fermentation pathway of $S$. stipitis strain ${ }^{4,14,15}$. Three key step enzymes in $S$. stipitis xylose fermentation pathway are (1) xylose reductase (EC.1.1.1.21), which converts xylose to xylitol using $\mathrm{NAD}(\mathrm{P}) \mathrm{H}$ as a cofactor ${ }^{16,17}$ (2) xylitol dehydrogenase (EC.1.1.1.9), which converts xylitol to xylulose using NAD as a cofactor ${ }^{18}$, and (3) xylulose kinase (EC 2.7.1.17), which converts xylulose to xylulose-5-phosphate ${ }^{19}$. Because under oxygen-limit condition which $S$. cerevisiae ferments glucose to ethanol, NAD becomes its limiting factor ${ }^{15}$. So ethanol production from xylose of the recombinant $S$. cerevisiae was low. Screening for NADH dependent xylose reductase which has high activity to replace the $\mathrm{NAD}(\mathrm{P}) \mathrm{H}$ dependent xylose reductase of $S$. stipitis will be useful in constructing a recombinant $S$. cerevisiae capable of fermenting xylose ${ }^{14,20,21}$. Digestive tract of herbivores contains microorganisms which can hydrolyse only cellulose in the lignocelluloses consumed to glucose ${ }^{22}$. So the existence of such xylose-utilizing yeasts in their faeces is expected. There is no previous report on the diversity of xylose-utilizing yeasts in digestive tract or faeces of herbivores in Thailand. The objective of this work is to isolate xylose-utilizing yeasts from herbivore faeces to screen for high efficient xylose fermenting and xylitol producing yeasts. Co-enzyme specificity of high activity xylose reductase enzymes of selected yeast was also determined.

\section{MATERIALS AND METHODS}

\section{Isolation of xylose utilizing yeasts}

Twenty-one herbivore faeces samples from 5 elephants, 1 goat, 2 giraffes, 1 macaque, 3 cows, 1 buffalo, 1 kangaroo, 2 zebras, 1 hog deer, 1 orlik, 1 antelope, 1 horse, and 1 barking deer were collected from various areas in Thailand (Table 1). The sample $0.5 \mathrm{~g}$ was enriched in $10 \mathrm{ml}$ of Yeast Nitrogen BaseXylose (YX) medium $(0.67 \%$ yeast nitrogen base and $5 \%$ D-xylose, w/v) supplemented with $0.02 \%$ chloramphenicol and $0.25 \%$ sodium propionate in $25 \times 150 \mathrm{~mm}$ test tube. The enriched samples were incubated at $30^{\circ} \mathrm{C}$ for 3-10 days, then spread on YX agar for isolation. Representative yeast colonies were selected, purified, kept on Yeast extract-Malt extract (YM) slant $(0.3 \%$ yeast extract, $0.3 \%$ malt extract, $0.5 \%$ peptone, $1 \%$ glucose and $1.5 \%$ agar, w/v) at $4{ }^{\circ} \mathrm{C}$ and stored for a long time in YM broth containing $10 \%$ $(\mathrm{w} / \mathrm{v})$ glycerol at $-80^{\circ} \mathrm{C}^{23}$.

\section{IDENTIFICATION METHODS}

\section{Phenotypic characterization}

Morphological, physiological and biochemical characteristics of isolated yeasts were examined according to Yarrow $^{29}$. Cells grown on 5\% malt extract agar, cornmeal agar or YM agar for 1 or 5 days were examined for ascospore formation under microscope. Sugar fermentation tests of D-galactose, D-glucose, lactose, maltose, D-raffinose, sucrose, trehalose and D-xylose were determined using $0.67 \%$ yeast nitrogen base solution containing 2\% sugar with Durham tube $^{30}$. Assimilation of carbon and nitrogen compounds were determined in liquid and solid medium, respectively. Nitrogen source tested were $\mathrm{KNO}_{3}$, $\mathrm{NaNO}_{2}$, ethylamine hydrochloride, cadaverine dihydrochloride, and lysine (Table 2). Capability to grow in $50 \%$ glucose and at $37^{\circ} \mathrm{C}$ were determined by using YM broth and incubated by using a heat block. Glucose/mineral medium without all vitamins was used to test for an ability to grow in the absence of vitamins. Sensitivity to cycloheximide was performed by using Bacto Yeast Nitrogen Base containing D-glucose and supplemented with $0.1 \%$ or $0.01 \%$ cycloheximide.

\section{RNA gene sequence and phylogenetic analysis}

The D1/D2 domain of large subunit ribosomal RNA gene ( $L S U$ rRNA gene) was amplified by PCR using the primers NL1 $\left(5^{\prime}\right.$-GCATATCAATAAGCGGAG GAA- $\left.3^{\prime}\right)$ and NL4 (5'-GGTCCGTGTTTCAAGAC GG-3' $)^{5,31}$. PCR product was purified by QIAquick purification kit (Qiagen K.K., Japan). The purified PCR product was sequenced by using ABI BigDye Terminator V3.1 Cycle Sequencing RR-100 kit, and ABI Model 3130xl DNA Analyser (Applied Biosystems, Foster City, California, USA). DNA Sequencing reaction was performed using the primers NL1 and NL4. Sequencing data was aligned by Chromas Pro software program (Technelysium Pty Ltd., Australia). The sequences were compared pairwise using a BLASTN search ${ }^{32}$ and were aligned with sequences of related species retrieved from GenBank using multiple alignment program CLUSTAL_X version $1.8^{33}$. The D1/D2 domain of $L S U$ rRNA gene sequence similarity of $99-100 \%$ to those of related species was identified as the same species ${ }^{5}$. Phylogenetic tree was constructed from an evolutionary distance data with Kimura's two-parameter correction ${ }^{34}$ using neighbour-joining method ${ }^{35}$. Topology of the phylogenetic tree was tested by performing 1000 replicates bootstrap resampling ${ }^{36}$. 
Table 1 Isolation and identification of xylose-utilizing yeasts based on D1/D2 domain of $L S U$ rRNA gene sequence.

\begin{tabular}{llll}
\hline Animal faeces/Province & Isolate no. & Similarity (\%) & Identification \\
\hline Elephant/Bangkok & $\mathrm{A} 1, \mathrm{~A} 2$ & 100 & C. tropicalis \\
Elephant/Nakhonratchasima & $\mathrm{A} 19$ & 100 & C. tropicalis \\
Elephant/Prachinburi & $\mathrm{B} 1$ & 100 & C. tropicalis \\
Goat/Bangkok & $\mathrm{A} 3$ & 100 & C. tropicalis \\
Giraffe/Bangkok & $\mathrm{A} 4$ & 100 & C. tropicalis \\
Giraffe/Nakhonratchasima & $\mathrm{A} 21$ & 100 & C. tropicalis \\
Cow/Khonkaen & $\mathrm{A} 6, \mathrm{~A} 26$ & 100 & C. tropicalis \\
Cow/Udonthani & $\mathrm{A} 9, \mathrm{~A} 10, \mathrm{~A} 11$ & C. tropicalis \\
Cow/Kalasin & $\mathrm{A} 12, \mathrm{~A} 13, \mathrm{~A} 15$ & 100 & C. tropicalis \\
Buffalo/Kalasin & $\mathrm{A} 7, \mathrm{~A} 8, \mathrm{~A} 16, \mathrm{~A} 17$ & 100 & C. tropicalis \\
Kangaroo/Nakhonratchasima & $\mathrm{A} 18$ & 100 & C. tropicalis \\
Hog deer/Nakhonratchasima & $\mathrm{A} 22$ & 100 & C. tropicalis \\
Antelope/Nakhonratchasima & $\mathrm{A} 23$ & 100 & C. tropicalis \\
Oryx/Nakhonratchasima & $\mathrm{A} 24$ & 100 & C. tropicalis \\
Horse/Nakhonratchasima & $\mathrm{A} 25$ & 100 & C. tropicalis \\
Barking deer/Bangkok & $\mathrm{A} 27, \mathrm{D} 1, \mathrm{E} 1$ & 100 & C. tropicalis \\
Zebra/Bangkok & $\mathrm{A} 28, \mathrm{I} 1$ & 100 & C. tropicalis \\
Zebra/Nakhonratchasima & $\mathrm{A} 20, \mathrm{M} 1$ & 100 & C. tropicalis \\
Macaque/Bangkok & $\mathrm{A} 5$ & 100 & C. tropicalis \\
Kangaroo/Bangkok & $\mathrm{L} 1$ & 100 & C. albicans \\
Macaque/Bangkok & $\mathrm{H} 1$ & 99 & P. terricola \\
Elephant/Ayutthaya & $\mathrm{E} 21, \mathrm{E} 24$ & 100 & T. mycotoxinivorans \\
Elephant/Ayutthaya & $\mathrm{E} 20$ & 100 & S. lactativora \\
Elephant/Nakhon Pathom & $\mathrm{E} 25$ & 99 & S. lactativora \\
Elephant/Ayutthaya & $\mathrm{E} 23$ & 99 & Z. meyerae \\
\hline
\end{tabular}

\section{Determination of ethanol and xylitol production}

Single colony of yeast isolates grown on $\mathrm{YX}$ agar, $\mathrm{pH} 5.0$ at $30^{\circ} \mathrm{C}, 48 \mathrm{~h}$ was inoculated into $\mathrm{YX}$ broth (50 $\mathrm{ml}$ in $250 \mathrm{ml}$ Erlenmeyer flask), incubated at $30{ }^{\circ} \mathrm{C}, 200 \mathrm{rpm}$ for $24 \mathrm{~h}$. The culture was transferred at $1 \%(\mathrm{v} / \mathrm{v})$ into fresh $\mathrm{YX}$ broth $(50 \mathrm{ml}$ in $250 \mathrm{ml}$ Erlenmeyer flask) and incubated at $30^{\circ} \mathrm{C}, 200 \mathrm{rpm}$ for $24 \mathrm{~h}$. The inoculum was inoculated at $10 \%(\mathrm{v} / \mathrm{v})$ into the same medium and incubated at $30^{\circ} \mathrm{C}, 200 \mathrm{rpm}$ for $24 \mathrm{~h}$. After centrifugation at $4{ }^{\circ} \mathrm{C}, 9793 \mathrm{~g}(10 \mathrm{~min})$, resultant supernatants were analysed for ethanol by gas chromatography ${ }^{37}$ and for xylitol by high performance liquid chromatography (HPLC) ${ }^{38}$. S. stipitis JCM $10742^{\mathrm{T}}$ (Japan Collection of Microorganisms, RIKEN BioResource Centre, Japan), which is a high efficient ethanolic xylose fermenting yeast, was used as a control.

Single colony of top seven high xylitol producing yeast selected was grown in modified media of Kurtzman and Dien ${ }^{39}$ (Yeast-Peptone-Xylose, YPX medium containing $1 \%$ yeast extract and $2 \%$ peptone, $6.6 \%$ xylose (w/v) and $\mathrm{pH} 5.0)$ at $30^{\circ} \mathrm{C}$ for $24 \mathrm{~h}$. Optical density at $660 \mathrm{~nm}(\mathrm{OD} 660 \mathrm{~nm})$ of the resultant culture was adjusted to 2.0, inoculated into fresh YPX medium (50 $\mathrm{ml}$ in $250 \mathrm{ml}$ Erlenmeyer flask) at $10 \%$ (v/v) and incubated at $30^{\circ} \mathrm{C}, 200 \mathrm{rpm}$ for $24-48 \mathrm{~h}$. The culture was centrifuged and obtained supernatant was analysed for ethanol and xylitol.

\section{Cloning of xylose reductase gene}

Xylose reductase gene ( $x y l$ ) was amplified directly from genomic DNA of $C$. tropicalis A26 by PCR method. Primers specific for $x y l$ were designed based on conserved amino acid sequences of xylose reductase of various yeast species. The primers were designated as XR_F [5'-GA(G/A)A(A/G)(A/G)TA(T/C) CC(A/T)GG(A/T)TTCTAC-3'] and XR_R [5'-CCT (G/A)TCCCA(T/G)GG(A/G)T(T/C)(G/A)TT(G/A) AATCT- $3^{\prime}$ ]. The amplification was performed by gradient PCR using Tag DNA Polymerase (Fermentas, California). The amplified product was purified by GeneJET Gel Extraction Kit (Fermentas, California) then ligated to pTZ57R/T vector (InsTAclone PCR cloning kit, Fermentas, California). The ligation reaction was transformed into Escherichia coli $\mathrm{DH} 5 \alpha$ competent cells using method described by Sambrook and Russell ${ }^{40}$. Colonies grown on LB agar containing $100 \mu \mathrm{g} / \mathrm{ml}$ ampicillin, $20 \mathrm{mg} / \mathrm{ml} \mathrm{X-Gal} \mathrm{and} 100 \mathrm{mM}$ 
ScienceAsia 39 (2013)

Table 2 Differential characteristics of yeasts isolated and their related type strains.

\begin{tabular}{|c|c|c|c|c|c|c|c|c|c|c|c|c|}
\hline Characteristics & 1 & $2^{\mathrm{a}}$ & 3 & $4^{\mathrm{a}}$ & 5 & $6^{\mathrm{b}}$ & 7 & $8^{c}$ & 9 & $10^{\mathrm{d}}$ & 11 & $12^{\mathrm{e}}$ \\
\hline \multicolumn{13}{|l|}{ Fermentation of: } \\
\hline Glucose & + & + & + & + & + & $+/ w$ & - & - & - & - & + & + \\
\hline Galactose & + & + & + & $\mathrm{v}$ & - & - & - & - & - & - & w & w \\
\hline Sucrose & + & $\mathrm{v}$ & + & $\mathrm{v}$ & - & - & - & - & - & - & w & w \\
\hline Maltose & + & + & + & + & - & - & - & - & - & - & - & - \\
\hline Lactose & - & - & + & - & - & - & - & - & - & - & - & - \\
\hline Raffinose & - & - & + & - & - & - & - & - & - & - & - & $\mathrm{w} / \mathrm{l}$ \\
\hline Trehalose & + & $+/ \mathrm{s}$ & + & $\mathrm{v}$ & - & - & - & - & - & - & $\mathrm{s}$ & $\mathrm{w} / \mathrm{l}$ \\
\hline \multicolumn{13}{|l|}{ Assimilation of: } \\
\hline Glucose & + & + & + & + & + & + & + & + & 1 & + & + & + \\
\hline Galactose & + & + & + & + & - & - & + & + & - & - & 1 & + \\
\hline L-Sorbose & $\mathrm{s}$ & $\mathrm{v}$ & $\mathrm{s}$ & $\mathrm{v}$ & - & - & $\mathrm{s}$ & + & 1 & + & 1 & + \\
\hline Sucrose & + & $\mathrm{v}$ & + & $\mathrm{v}$ & - & - & + & + & - & - & 1 & + \\
\hline Maltose & + & + & + & + & - & - & + & + & - & - & 1 & + \\
\hline Cellobiose & $\mathrm{w}$ & $+/ 1$ & - & - & - & - & + & + & - & - & 1 & + \\
\hline Trehalose & + & + & + & $\mathrm{v}$ & - & - & + & + & - & - & + & + \\
\hline Lactose & - & - & - & - & - & - & + & + & - & - & - & - \\
\hline Melibiose & - & - & - & $\mathrm{v}$ & - & - & 1 & + & - & - & - & - \\
\hline Raffinose & - & - & - & - & - & - & 1 & + & - & - & 1 & + \\
\hline Melezitose & + & $\mathrm{v}$ & $\mathrm{s}$ & - & - & - & w & + & - & - & 1 & $\mathrm{v}$ \\
\hline Inulin & $\mathrm{w}$ & - & $\mathrm{s}$ & - & - & - & - & + & $\mathrm{w}$ & - & - & - \\
\hline Soluble starch & + & + & 1 & + & - & - & + & + & - & - & 1 & + \\
\hline D-Xylose & + & + & + & + & - & - & + & + & 1 & + & + & + \\
\hline L-Arabinose & - & - & - & $\mathrm{v}$ & - & - & + & + & - & - & + & + \\
\hline D-Arabinose & - & - & - & $\mathrm{v}$ & - & - & - & + & - & - & - & - \\
\hline D-Ribose & - & $-/ 1$ & - & $\mathrm{v}$ & - & - & + & + & - & $\mathrm{v}$ & - & $\mathrm{v}$ \\
\hline L-Rhamnose & - & - & - & - & - & - & 1 & + & - & - & + & + \\
\hline Ethanol & + & + & + & + & + & + & + & + & 1 & + & + & + \\
\hline Glycerol & - & $\mathrm{v}$ & - & $\mathrm{v}$ & + & + & + & + & + & + & + & + \\
\hline Erythritol & - & - & - & - & - & - & - & w & - & - & - & - \\
\hline Ribitol & + & $+/ 1$ & + & + & - & - & $\mathrm{s}$ & - & + & + & 1 & + \\
\hline Galactitol & - & - & - & - & - & - & - & + & - & - & + & + \\
\hline D-Mannitol & + & + & + & + & - & - & 1 & + & 1 & $\mathrm{v}$ & + & + \\
\hline D-Glucitol & + & + & + & $\mathrm{v}$ & - & - & 1 & + & 1 & $\mathrm{v}$ & $\mathrm{s}$ & + \\
\hline$\alpha$-Methyl-D-glucoside & + & $\mathrm{v}$ & + & $\mathrm{v}$ & - & - & 1 & + & - & - & - & - \\
\hline Salicin & $\mathrm{v}$ & $\mathrm{v}$ & $\mathrm{s}$ & - & - & - & 1 & + & - & - & + & + \\
\hline DL-Lactate & + & $\mathrm{v}$ & + & + & 1 & - & + & & 1 & + & $\mathrm{w} /-$ & - \\
\hline Succinate & + & + & + & + & + & + & + & + & 1 & + & + & + \\
\hline Citrate & + & $+/ 1$ & 1 & + & + & w/- & + & + & - & - & $\mathrm{s}$ & + \\
\hline Inositol & - & - & - & - & - & - & 1 & + & 1 & + & + & + \\
\hline 2-Keto-D-gluconate & + & + & + & + & - & - & + & + & - & - & - & - \\
\hline 5-Keto-D-gluconate & + & + & 1 & $\mathrm{n}$ & - & - & + & + & - & $\mathrm{n}$ & $\mathrm{s}$ & $\mathrm{n}$ \\
\hline Nitrite & - & - & - & - & - & $\mathrm{n}$ & - & - & - & $\mathrm{n}$ & - & - \\
\hline Cadaverine & + & + & + & + & + & $\mathrm{n}$ & + & w & - & - & + & + \\
\hline L-Lysine & + & + & + & + & + & $\mathrm{n}$ & + & + & + & + & & - \\
\hline Ethylamine & + & + & + & + & + & $\mathrm{n}$ & + & w & + & + & + & + \\
\hline \multicolumn{13}{|l|}{ Growth in: } \\
\hline Vitamin-free & 1 & $\mathrm{v}$ & + & $\mathrm{v}$ & - & - & $\mathrm{w}$ & - & $\mathrm{w}$ & - & - & - \\
\hline $50 \%$ Glucose & - & $+/ 1$ & + & $\mathrm{v}$ & - & $\mathrm{n}$ & + & - & - & - & + & - \\
\hline $10 \% \mathrm{NaCl} / 5 \%$ Glucose & + & 1 & + & $\mathrm{v}$ & - & - & + & - & - & $\mathrm{n}$ & + & $\mathrm{n}$ \\
\hline $0.01 \%$ Cycloheximide & + & + & + & + & - & $\mathrm{n}$ & + & + & 1 & + & 1 & + \\
\hline $0.1 \%$ Cycloheximide & + & + & + & + & - & $\mathrm{n}$ & + & + & 1 & $\mathrm{n}$ & 1 & + \\
\hline Growth at $37^{\circ} \mathrm{C}$ & + & + & + & + & + & $\mathrm{v}$ & + & + & 1 & + & + & + \\
\hline Starch formation & - & - & - & - & - & - & - & - & - & $\mathrm{n}$ & - & - \\
\hline Urease & - & - & - & $\mathrm{n}$ & - & $\mathrm{n}$ & + & + & - & $\mathrm{n}$ & - & - \\
\hline
\end{tabular}

1, C. tropicalis (32 isolates); 2, C. tropicalis NRRL Y-12968 ${ }^{\mathrm{T}} ; 3$, C. albicans (1 isolate, L1); 4, C. albicans NRRL Y-17909 ${ }^{\mathrm{T}} ; 5$, P. terricola (1 isolate, H1); 6, P. terricola YB-4310 $;$ 7, T. mycotoxinivorans (2 isolates); 8 , T. mycotoxinivorans $\mathrm{HB} 1175^{\mathrm{T}}$; 9, S. lactativora (2 isolates); 10, S. lactativora NRRL Y-11591 ${ }^{\mathrm{T}}$; 11 , Z. meyerae (1 isolate, ELP23); 12, Z. meyerae Y-17319 ${ }^{\mathrm{T}}$. +, positive; -, negative; 1, latent (longer than 7 days), s, slow; w, weak; v, variable; $n$, no data.

${ }^{a}$ Data from Lachance et $\mathrm{al}^{24}$.

${ }^{\mathrm{b}}$ Data from Kurtzman ${ }^{25}$.

${ }^{c}$ Data from Molnar et $\mathrm{al}^{26}$.

${ }^{\mathrm{d}}$ Data from Rodrigues et $\mathrm{al}^{27}$.

e Data from Smith et al ${ }^{28}$. 
IPTG after incubation at $37^{\circ} \mathrm{C}$ for $24 \mathrm{~h}$ were collected. Clones harbouring the $x y l$ were determined by colony PCR. Confirmation of the $x y l$ gene was performed by DNA sequence analysis.

\section{Analytical procedures}

Ethanol was analysed by gas chromatography (Hewlett-Packard, HP 5890 Series; USA) using Parapak QS (Carbowax 20 M) column and flame ionization detector (FID, $150^{\circ} \mathrm{C}$ ). Oven temperature was $175^{\circ} \mathrm{C}$ and using helium at $35 \mathrm{ml} / \mathrm{min}$ flow rate as carrier gas, Xylitol was quantified by HPLC (Varian, Prostar, USA) using Lichrospher100 $\mathrm{NH}_{2}$ (4$250 \mathrm{~mm}$ ) column (Merck, Germany) and evaporative light scattering detector. (Alltech, USA). Mobile phase was acetonitide:water (91:9) at $1.5 \mathrm{ml} / \mathrm{min}$ flow rate.

\section{RESULTS AND DISCUSSION}

\section{Identification of yeast isolates}

Thirty-nine xylose-utilizing yeasts were assigned to ascomycetous (37 isolates), and basidiomycetous (2 isolate) yeasts. The 37 yeast isolates were identified as C. tropicalis (32 isolates), C. albicans (1 isolate), Pichia terricola (1 isolate), Sporopachydermia lactativora (2 isolates) and Zygoascus meyerae (1 isolate), and the 2 basidiomycetous yeasts were identified as Trichosporon mycotoxinivorans based on their phenotypic characteristics and D1/D2 domain of $L S U$ rRNA gene sequence analysis (Fig. 1 and Table 2).

The majority of the yeast isolates (32 isolates) had the following characteristics: globose cell shape, formed pseudohyphae, consisted of cylindrical cells shape, and branched chains. All isolates fermented glucose, galactose, sucrose, maltose, and trehalose. They assimilated sucrose, L-sorbose (slow), cellobiose (weak), melezitose, inulin (weak), ribitol, $\alpha$-methyl-D-glucoside, citrate (latent), grew on vitamin-free medium, $10 \% \mathrm{NaCl}$ with $5 \%$ glucose, and grew on YM medium at $37^{\circ} \mathrm{C}$. They did not grow on $50 \%$ glucose which was different from $C$. tropicalis NRRL Y-12968 ${ }^{\mathrm{T}}$ (Table 2). They were clustered within C. tropicalis NRRL Y-12968 ${ }^{\mathrm{T}}$ (Fig. 1). Their D1/D2 LSU rRNA gene sequences were 100\% similarity to C. tropicalis NRRL Y-12968 ${ }^{\mathrm{T}}$ (Table 1). Therefore, they were identified as $C$. tropicalis $^{24}$.

Isolate L1 had globose cell shape and formed branched pseudohyphae. It fermented galactose, sucrose, lactose, and raffinose, assimilated L-sorbose (slow), sucrose, trehalose, melezitose (slow), inulin (slow), glucitol, $\alpha$-methyl-D-glucoside, salicin (slow), and citrate (latent), but did not assimilate melibiose, L-arabinose, D-arabinose and D-ribose, grew on vitamin free medium, $50 \%$ glucose, $10 \%$ $\mathrm{NaCl}$ with $5 \%$ glucose (Table 2). C. albicans has variable characteristic ${ }^{4}$. The isolate was related to C. albicans (Fig. 1) and was clustered in Lodderomyces-Spathaspora clade. It showed $99 \%$ D1/D2 LSU rRNA gene sequence similarity to C. albicans NRRLY- $17909^{\mathrm{T}}$ with 2 nucleotides difference (Table 1). Therefore, it was identified as C. albicans $^{24}$.

Isolate $\mathrm{H} 1$ fermented glucose, grew on $\mathrm{YM}$ medium at $37^{\circ} \mathrm{C}$, assimilated DL-lactate, citrate, cadaverine, L-lysine, and ethylamine. It showed almost the same phenotypic characteristics as P. terricola YB- $4310^{\mathrm{T}}$ (Table 2). The isolate was nearest with P. terricola $\mathrm{YB}-4310^{\mathrm{T}}$ based on $100 \% \mathrm{D} 1 / \mathrm{D} 2 \mathrm{LSU}$ rRNA gene sequence similarity (Fig. 1, Table 1). It belonged to $P$. membranifaciens clade which was transferred from Issatchenkia and moved back to Pichia $^{41}$. Therefore it was identified as P. terricola ${ }^{25}$.

Isolates E21 and E24 showed various cell morphology (i.e., unicellular, yeast-like, pseudohyphal, and hyphal). They assimilated L-sorbose (slow), melibiose (latent), raffinose (latent), melezitose (weak), rhamnose (latent), ribose (slow), mannitol (latent), glucitol (latent), $\alpha$-methyl-D-glucoside (latent), salicin (latent), and inositol (latent), but did not assimilate inulin, D-arabitol, erythritol, or galactitol. They grew on vitamin free medium (weak), $50 \%$ glucose, and $10 \% \mathrm{NaCl}$ with $5 \%$ glucose (Table 2). They were related to T. mycotoxinivorans $\mathrm{HB} 1175^{\mathrm{T}}$ based on 100\% D1/D2 LSU rRNA gene sequence similarity (Fig. 1 and Table 1). Therefore, they were identified as T. mycotoxinivorans ${ }^{26}$.

Isolates, E20 and E25, assimilated glucose (latent), L-sorbose (latent), inulin (weak), D-xylose (latent), ethanol (latent), ribitol, mannitol (latent), salicin (latent), and grew on vitamin free medium (weak). They neither fermented sugars nor assimilated cadaverine (Table 2). They were related to Sporopachydermia lactativora NRRL Y-11591 ${ }^{\mathrm{T}}$ based on $99 \%$ D1/D2 LSU rRNA gene sequence similarity (Fig. 1, Table 1). They were identified as S. lactativora ${ }^{27}$.

Isolate E23 assimilated galactose, L-sorbose, sucrose, maltose, cellobiose, raffinose, melezitose, soluble starch, ribitol, and $0.1 \%$ cycloheximide after 7 days (latent). It neither fermented raffinose nor assimilated ribose (Table 2). The isolate was nearest to Z. meyerae $\mathrm{Y}-17913^{\mathrm{T}}$ (Fig. 1). The D1/D2 LSU rRNA gene sequence of this isolate was $100 \%$ similarity to Z. meyerae $\mathrm{Y}-17913^{\mathrm{T}}$ with only one base substitution (Table 1). Therefore, the isolate was identified as Z. meyerae ${ }^{28}$. 


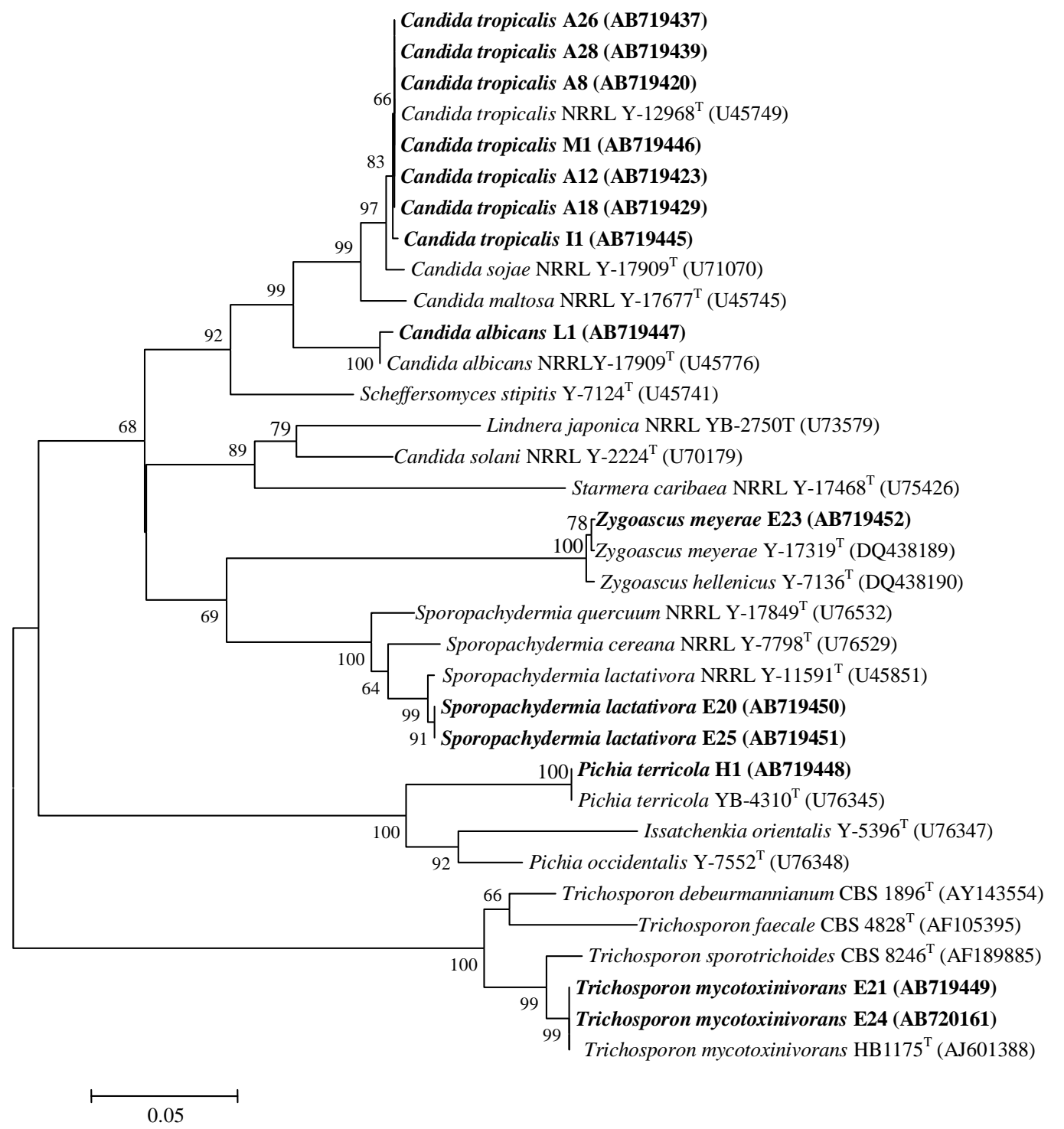

Fig. 1 Phylogenetic tree of xylose assimilation yeasts constructed by the neighbour-joining method based on D1/D2 domain of $L S U$ rRNA gene sequences. The numbers represent the percentages from 1000 replicates bootstrap resampling (frequencies less than $50 \%$ are not shown).

\section{Ethanol and xylitol production}

The 39 xylose-utilizing yeasts isolated were cultured in YX medium. It was found that 37 isolates produced ethanol (0.0016-0.0895 g/l) after $24 \mathrm{~h}$ (data not shown), 2 isolates (P. terricola $\mathrm{H} 1$ and $C$. albicans L1) could not produce ethanol. Z. meyerae E23 produced the highest ethanol at 2.04 and $3.61 \mathrm{~g} / \mathrm{l}$ after 48 and $72 \mathrm{~h}$ (data not shown), respectively. For xylitol production, 36 out of 39 xylose-utilizing yeasts isolated produced xylitol in the range of $0.03-43 \mathrm{~g} / \mathrm{l}$ (data not shown). Seven $C$. tropicalis isolates, A8,
A12, A18, A26, A28, I1, and M1 which produced xylitol more than $30 \mathrm{~g} / \mathrm{l}$ and the Z. meyerae E23 which produced the highest ethanol were selected for further studied, since their xylose reductase was expected to have high activity. In YPX medium, the eight selected isolates were found to produce ethanol (0.012-0.017 g/g xylose). Prolongation of the incubation period from $24-48 \mathrm{~h}$ only increased ethanol production by Z. meyerae E23 from $0.017-0.08 \mathrm{~g} / \mathrm{g}$ xylose. Xylose consumption rate of $Z$. meyerae E23 was comparatively low during the first $24 \mathrm{~h}$ (Table 3 ). In this study, our isolate produced ethanol from xylose 
Table 3 Growth and xylose fermentation parameters of selected isolates.

\begin{tabular}{lcccccccc}
\hline Strains & $\mathrm{Y}_{\mathrm{cm} / \mathrm{s}}(\mathrm{g} / \mathrm{g})$ & $\mathrm{Q}_{\mathrm{s}}\left(\mathrm{g}^{-1} \mathrm{~h}^{-1}\right)$ & $\mathrm{C}_{\mathrm{xl}}(\mathrm{g} / \mathrm{l})$ & $\mathrm{C}_{\mathrm{e}}(\mathrm{g} / \mathrm{l})$ & $\mathrm{Q}_{\mathrm{xl}}\left(\mathrm{g} \mathrm{l}^{-1} \mathrm{~h}^{-1}\right)$ & $\mathrm{Q}_{\mathrm{e}}\left(\mathrm{g} \mathrm{l}^{-1} \mathrm{~h}^{-1}\right)$ & $\mathrm{Y}_{\mathrm{xl} / \mathrm{s}}(\mathrm{g} / \mathrm{g})$ & $\mathrm{Y}_{\mathrm{e} / \mathrm{s}}(\mathrm{g} / \mathrm{g})$ \\
\hline A8 & 0.11 & 2.07 & 33.69 & 0.87 & 1.40 & 0.04 & 0.68 & 0.017 \\
$\mathrm{~A} 26$ & 0.08 & 2.56 & 43.79 & 0.73 & 1.82 & 0.03 & 0.71 & 0.012 \\
M1 & 0.08 & 2.43 & 39.59 & 0.68 & 1.65 & 0.03 & 0.68 & 0.012 \\
A12 & 0.09 & 2.25 & 38.33 & 0.81 & 1.60 & 0.03 & 0.71 & 0.015 \\
$\mathrm{I} 1$ & 0.11 & 2.03 & 33.83 & 0.72 & 1.41 & 0.03 & 0.69 & 0.015 \\
A28 & 0.09 & 2.44 & 40.66 & 0.87 & 1.69 & 0.04 & 0.69 & 0.015 \\
A18 & 0.11 & 2.23 & 27.36 & 0.83 & 1.14 & 0.03 & 0.51 & 0.016 \\
E23 & 0.28 & 1.05 & 7.89 & 0.42 & 0.33 & 0.02 & 0.31 & 0.017 \\
JCM 10742 & 0.11 & 2.73 & 0.96 & 18.66 & 0.04 & 0.78 & 0.01 & 0.285 \\
\hline
\end{tabular}

The values presented are averages of duplicate experiments.

$\mathrm{Y}_{\mathrm{cm} / \mathrm{s}}$, biomass yield (g dry mass/g D-xylose consumed); $\mathrm{Q}_{\mathrm{s}}$, volumetric xylose consumption rate $\left(\mathrm{g}^{-1} \mathrm{~h}^{-1}\right) ; \mathrm{C}_{\mathrm{xl}}$, maximum volumetric xylitol concentration observed $(\mathrm{g} / \mathrm{l}) ; \mathrm{C}_{\mathrm{e}}$, maximum volumetric ethanol concentration observed $(\mathrm{g} / \mathrm{l}) ; \mathrm{Q}_{\mathrm{xl}}$, volumetric xylitol production rate $\left(\mathrm{g} \mathrm{l}^{-1} \mathrm{~h}^{-1}\right) ; \mathrm{Q}_{\mathrm{e}}$, volumetric ethanol production rate $\left(\mathrm{g}^{-1} \mathrm{~h}^{-1}\right) ; \mathrm{Y}_{\mathrm{xl} / \mathrm{s}}$, xylitol yield (g xylitol/g D-xylose consumed); $\mathrm{Y}_{\mathrm{e} / \mathrm{s}}$, ethanol yield (g ethanol/g D-xylose consumed).

with a small amount compared to S. stipitis JCM $10742^{\mathrm{T}}(0.285 \mathrm{~g} / \mathrm{g}$ xylose), Kluyveromyces marxianus $^{42}(0.28 \mathrm{~g} / \mathrm{g}$ xylose), or Spathaspora passalidarum $^{43}(0.4 \mathrm{~g} / \mathrm{g}$ xylose) which have been improved for ethanol production. However, Z. meyerae E23 was the first report to produce ethanol and further study is required. For xylitol production it was found that maximum xylitol $(0.71 \mathrm{~g} / \mathrm{g}$ xylose) was produced by C. tropicalis A26 and A12. Since C. tropicalis A26 produced higher xylitol $(43.79 \mathrm{~g} / 1)$ than $C$. tropicalis A12 (38.33 g/l). Therefore, C. tropicalis A26 was selected for further studies. P. miso, C. guilliermondii, C. tropicalis, C. mogii, C. maltosa, and H. polymorpha were reported on xylitol production in the range of $0.50-0.65 \mathrm{~g} / \mathrm{g}^{44-48}$. Guo et al ${ }^{49}$ reported that $C$. guilliermondii Xu280 and C. maltosa Xu316 produced xylitol $0.73 \mathrm{~g} / \mathrm{g}$ and $0.70 \mathrm{~g} / \mathrm{g}$, respectively, when they were grown in YPX medium supplemented with $50 \mathrm{~g} / \mathrm{l}$ xylose. Sreenivas et $\mathrm{al}^{50}$ reported that YS54 was the best xylitol producer $(0.58 \mathrm{~g} / \mathrm{g})$ among 35 yeasts isolated from gut of coleoptera insects. C. tropicalis produced xylitol by cell-recycle fermentation in a cross flow membrane bioreactor ${ }^{23,38}$. Xylitol yields of $0.82 \mathrm{~g} / \mathrm{g}$ xylose ${ }^{38}$ and $0.85 \mathrm{~g} / \mathrm{g}$ xylose ${ }^{23}$ were reported.

\section{Cloning of $\boldsymbol{C}$. tropicalis A26 xylose reductase gene}

Several yeasts such as Candida, Pachysolen and Debaryomyces reduced xylose to xylitol by $\mathrm{NAD}(\mathrm{P}) \mathrm{H}$ dependent xylose reductase (XR) ${ }^{46,51,52}$. The xylose reductase is encoded from the xylose reductase gene $(x y l)$. Xylose reductase gene $(x y l)$ of $C$. tropicalis A26 was amplified from genomic DNA by gradient PCR using designed primers; XR-F and XR-R. Desired amplicon of $540 \mathrm{bp}$ was obtained at annealing temperature range of $45-55^{\circ} \mathrm{C}$. The obtained PCR

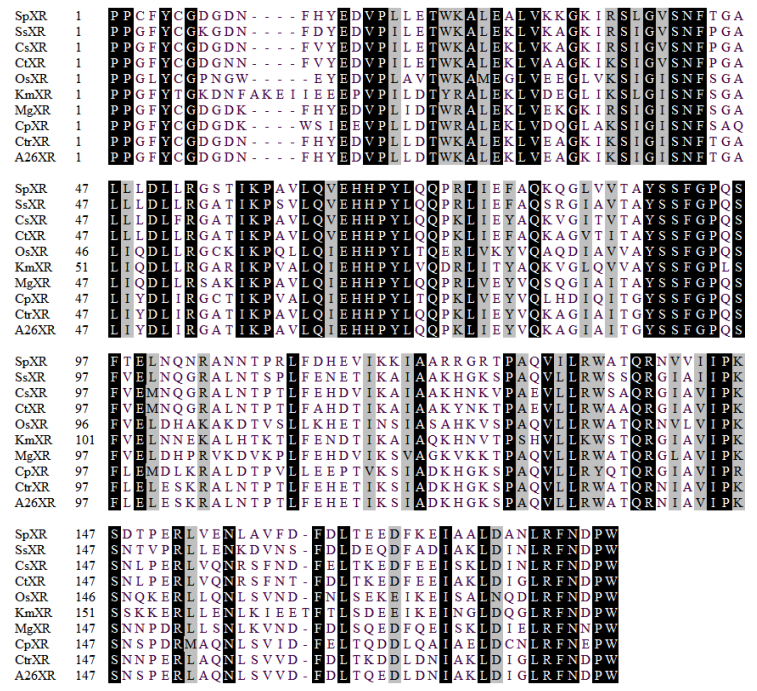

Fig. 2 Alignment of partial amino acid sequences of xylose reductase from $C$. tropicalis A26 and other yeast strains. A26XR, C. tropicalis A26; SpXR, Spathaspora passalidarum, (EGW32258); SsXR, Scheffersomyces stipitis (CAA42072); CsXR, C. shehatae (ABK35120); CtXR, C. tenuis (AAC25601); OsXR, Ogataea siamensis (ACN78427); KmXR, Kluyveromyces marxianus (ADV91498); MgXR from Meyerozyma guilliermondii (AAD09330); CpXR, C. parapsilosis (AAO91803); CtrXR, C. tropicalis (ABX60132); The underlined conserved sequences were used to design degenerated primers for the $x y l$ cloning. Rectangle box indicates $\mathrm{NAD}(\mathrm{P}) \mathrm{H}$ coenzyme use $^{43,45,47}$.

product was then purified and ligated to $\mathrm{pTZ}$ T7R/T vector. After transformation into $E$. coil $\mathrm{DH} 5 \alpha$ 
Table 4 Comparison of amino acid sequence similarity and identity between $C$. tropicalis A26 xylose reductase and those of others.

\begin{tabular}{lcccccccccc}
\hline Code & CtrXR & SpXR & SsXR & CsXR & CtXR & OsXR & KmXR & MgXR & CpXR & A26XR \\
\hline CtrXR & & 72.3 & 74.3 & 77.5 & 78.5 & 64.4 & 59.2 & 74.9 & 75.4 & 98.4 \\
SpXR & 83.8 & & 75.4 & 74.9 & 74.3 & 59.2 & 55.5 & 67.5 & 56.5 & 72.8 \\
SsXR & 82.7 & 83.8 & & 81.2 & 79.1 & 58.6 & 60.2 & 69.6 & 60.7 & 74.4 \\
CsXR & 86.4 & 84.8 & 87.4 & & 91.1 & 57.6 & 61.3 & 70.7 & 63.9 & 77.5 \\
CtXR & 86.9 & 82.2 & 86.9 & 94.8 & & 56.5 & 61.3 & 69.1 & 61.8 & 78.5 \\
OsXR & 73.3 & 71.2 & 69.6 & 68.6 & 69.1 & & 59.2 & 66.5 & 59.7 & 64.4 \\
KmXR & 71.7 & 70.7 & 72.3 & 71.2 & 71.7 & 70.7 & & 59.2 & 54.5 & 59.7 \\
MgXR & 83.8 & 80.6 & 80.6 & 81.2 & 80.1 & 74.3 & 70.2 & & 66.0 & 75.4 \\
CpXR & 82.7 & 73.8 & 73.8 & 74.9 & 73.8 & 69.1 & 67.5 & 75.4 & & 75.9 \\
A26XR & 99.0 & 84.3 & 84.2 & 85.2 & 86.4 & 72.8 & 71.7 & 83.8 & 83.8 & \\
\hline
\end{tabular}

Numbers on the lower-left half are Similarity (\%). Numbers on the upper-right half are Identity (\%). Similarity Codes are the same as in Fig. 2. Identity means the same amino acid; Similarity means amino acid in the same groups.

host, the putative positive clones were determined by colony PCR. Nucleotide sequence of an insert DNA extracted from a putative positive clone was analysed. Partial sequence of $x y l$ clone which consisted of 558 nucleotides coding for 186 amino acids is shown in Fig. 2. Comparison the obtained partial amino acid sequences of $C$. tropicalis A26 to those of other yeast strains in Gene Bank database revealed that $C$. tropicalis A26 xylose reductase possessed 98.4\%, 78.5\%, and $77.5 \%$ identity and $99.0 \%, 86.4 \%$, and $85.9 \%$ similarity to xylose reductase of $\mathrm{CtrXR}$ of $C$. tropicalis (ABX60132C), CtXR of C. tenuis (AAC25601), and CsXR of $C$. shehatae (ABK35120), respectively (Table 4). Amino acid sequence alignment of the partial xylose reductase revealed tetra amino acid motif (Ile-Pro-Lys-Ser), an NAD(P)H species motif ${ }^{14,17,53}$ (Fig. 2). The motif was well-conserved among xylose reductase of several yeast strains except for CpXR of $C$. parapsilosis which Lys residue was substituted with Arg and resulting in preferentially used NADH as coenzyme $^{54}$. The CtrXR that showed the highest sequence homology to those of A26XR was previously reported as NADPH-specific $\mathrm{XR}^{14,17}$. This result suggested that xylose reductase of $C$. tropicalis A26 might specify for an NADPH coenzyme, however, the expression of the $x y l$ clone including the characterization of xylose reductase expressed is required.

Acknowledgements: This study was supported by the Thailand Research Fund for a 2008 Royal Golden Jubilee $\mathrm{Ph}$.D. Programme as a scholarship to W.L. and Integrated Innovation Academic Centre: IIAC Chulalongkorn University Centenary Academic Development Project and the Higher Education Research Promotion and National Research University Project of Thailand, Office of the Higher Education Commission (CU56-EN13).

\section{REFERENCES}

1. Lee J (1997) Biological conversion of lignocellulosic biomass to ethanol. J Biotechnol 56, 1-24.

2. Jeffries TW, Alexander MA (1990) Production of ethanol from xylose by Candida shehatae grown under continuous or fed-batch condition. In: Kent Kirk T, Chang HM (eds) Biotechnology in Pulp and Paper Manufacture, Butterworth-Heineman, USA, pp 311-21.

3. Dien BS, Cotta MA, Jeffries TW (2003) Bacteria engineered for fuel ethanol production current status. Appl Microbiol Biotechnol 63, 258-66.

4. Byron CHC, Hung L (2007) Genetic improvement of Saccharomyces cerevisiae for xylose fermentation. Biotechnol Adv 25, 425-41.

5. Kutzman CP, Robnett CJ (1998) Identification and phylogeny of ascomycetous yeasts from analysis of nuclear large subunit (26S) ribosomal DNA partial sequences. Antonie Leeuwenhoek 73, 331-71.

6. Barnett JA, Payne RW, Yarrow D (2000) Yeast Characteristics and Identification, 3rd edn, Cambridge Univ press, UK.

7. Nitiyon S, Boonmak C, Am-In S, Jindamorakot S, Kawasaki H, Yongmanitchai W, Limtong S (2011) Candida saraburiensis sp. nov. and Candida prachuapensis sp. nov., xylose-utilizing yeast species isolated in Thailand. Int J Syst Evol Microbiol 61, 462-8.

8. Cadete RM, Santos RO, Melo MA, Mouro A, Goncaleves DL, Stambuk BU, Gomes FCO, Lanchance MA, Rosa CA (2009) Spathaspora arboratiae sp. Nov., a xylose-fermentating yeast species isolated from rooting wood in Brazil. FEMS Yeast Res 9, 1338-42.

9. Jefferies TW, Kurtzman CP (1994) Strain selection, taxonomy, and genetics of xylose-fermenting yeasts. Enzym Microb Tech 16, 922-32.

10. Nguyen NH, Suh SO, Marshall CJ, Blackwell M (2006) Morphological and ecological similarities: wood- 
boring beetles associated with novel xylose-fermenting yeasts, Spathaspora passalidarum gen. sp. nov. and Candida jeffriesii sp. nov. Mycol Res 110, 1232-41.

11. Jeffries TW (2006) Engineering yeast for xylose metabolism. Curr Opin Biotechnol 17, 320-6.

12. Taniguchi M, Itaya T, Tohma T, Fujii M (1997) Ethanol production from a mixture of glucose and xylose by a novel co-culture system with two fermentors and two microfiltration modules. J Ferment Bioeng 84, 59-64.

13. Agbogbo FK, Coward-Kelly G, Torry-Smith M, Wenger KS (2006) Fermentation of glucose/xylose mixture using Pichia stipitis. Process Biochem 41, 2333-6.

14. Watanabe S, Saleh AA, Pack SP, Annaluru N, Kodaki T, Makino K (2007) Ethanol production from xylose by recombinant Saccharomyces cerevisiae expressing protein engineered NADH-preferring xylose reductase from Pichia stipitis. Microbiology 153, 3044-54.

15. Jefferies TW, Jin YS (2004) Metabolic engineering for improved fermentation of pentoses by yeasts. Appl Biochem Biotechnol 63, 495-509.

16. Verduyn C, Van Kleef R, Frank J, Schreuder H, van Dijken VP, Scheffers WA (1985) Properties of the $\mathrm{NAD}(\mathrm{P}) \mathrm{H}-\mathrm{dependent}$ xylose reductase from the xylose-fermenting yeast. Pichia stipitis. Biochem $J$ 226, 669-77.

17. Zhan B, Zhang L, Wang D, Gao X, Hong J (2011) Identification of xylose reductase gene in the xylose metabolic pathway of Kluyveromyces marxianus NBRC177. J Ind Microbiol Biotechnol 38, 2001-11.

18. Rizzi M, Harwart K, Erlemann P, Buithanh NA, Dellweg H (1989) Purification andproperties of the NAD ${ }^{+}$ xylitol dehydrogenase from the yeast Pichia stipitis. J Ferment Bioeng 67, 20-4.

19. Jeppsson H, Yu S, Hahn-Hagerdal B (1996) Xylulose and glucose fermentation by Saccharomyces cerevisiae in chemostat culture. Appl Environ Microbiol 62, 1705-9.

20. Bengtsson O, Hahn-Hägerdal B, Gorwa-Grauslund MF (2009) Xylose reductase from Pichia stipitis with altered coenzyme preference improves ethanolic $x y-$ lose fermentation by recombinant Saccharomyces cerevisiae. Biotechnol Biofuels 2, 1-10.

21. Van Vleet JH, Jeffries TW (2009) Yeast metabolic engineering for hemicellulosic ethanol production. Curr Opin Biotechnol 20, 300-6.

22. Urubschurov V, Janczyk P, Pieper R, Souffrant WB (2008) Biological diversity of yeasts in the gastrointestinal tract of weaned piglets kept under different farm conditions. FEMS Yeast Res 8, 1349-56.

23. Kwon SG, Park SW, Oh DK (2006) Increase of xylitol productivity by cell-recycle fermentation of Candida tropicalis using submerged membrane bioreactor. J Biosci Bioeng 101, 13-8.

24. Lachance MA, Boekhout GS, Fell JW, Kurtzman CP (1923) Candida Berkhout. In: Kurtzman CP, Fell JW, Boekhout GS (eds) The Yeasts, a Taxonomic Study, 5th edn, Elsevier, Amsterdam, pp 987-1278.

25. Kurtzman CP (1998) Pichia E.C. Hansen emend. Kurtzman. In: Kurtzman CP, Fell JW (eds) The Yeasts, A Taxonomic Study, 4th edn, Elsevier, Amsterdam, pp 273-352.

26. Molnar O, Schatzmayr G, Fuchs E, Prillinger H (2004) Trichosporon mycotoxinivorans sp. nov., a new yeast species useful in biological detoxification of various mycotoxins. Syst Appl Microbiol 27, 661-71.

27. Rodrigues de Miranda L (1984) Sporopachydermia Rodrigues de Miranda. In: Kreger-van Rij NJW (ed) The Yeasts, A Taxonomic Study, 3rd edn, Elsevier, Amsterdam, pp 427-30.

28. Smith MTh Robert V, Poot GA,Epping W, de Cock AWAM (2005) Taxonomy and phylogeny of the ascomycetous yeast genus Zygoascus, with proposal of Zygoascus meyerae sp. nov. and related anamorphic varieties. Int J Syst Evol Microbiol 55, 1353-63.

29. Yarrow D (1998) Methods for the isolation, maintenance and identification of yeasts. In: Kurtzman CP, Fell JW (eds) The Yeasts, a Taxonomic Study, 4th edn, Elsevier, Amsterdam, pp 77-100.

30. Van der Walt JP (1957) Three new sporogenous yeasts from soil. Antonie Leeuwenhoek 23, 23-9.

31. O'Donnell K (1993) Fusarium and its near relatives. In: Reynolds DR, Taylor JW (eds) The Fungal Holomorph - Mitotic, Meiotic and Pleomorphic Speciation in Fungal Systematics. Wallingford, UK, pp 225-33.

32. Altschul SF, Madden TL, Schäffer AA, Zhang J, Zhang Z, Miler W, Lipman DJ (1997) Gapped BLAST and PSI-BLAST: a new generation of protein database search programs. Nucleic Acids Res 25, 3389-402.

33. Thompson JD, Gibson TJ, Pleniak F, Jeanmougin F, Higgins DG (1997) The CLUSTAL_X windows interface: flexible strategies for multiple sequence alignment aided by quality analysis tools. Nucleic Acids Res 25, 4876-82.

34. Kimura M (1980) A simple method for estimating evolutionary rate of base substitutions through comparative studies of nucleotide sequences. J Mol Evol 16, 111-20.

35. Saitou N, Nei M (1987) The neighbor-joining method: a new method for reconstructing phylogenetic tree. $\mathrm{Mol}$ Biol Evol 4, 406-25.

36. Felsenstein J (1988) Phylogenic from molecular sequences: inference and reliability. Аnпи Rev Genet 22, 521-65.

37. Akaracharanya A, Kesornsit J, Leepipatpiboon N, Srinorakutara T, Kitpreechavanich V, Tolieng V (2011) Evaluation of the waste from cassava starch production as a substrate for ethanol fermentation by Saccharomyces cerevisiae. Ann Microbiol 61, 431-6.

38. Choi JH, Moon KH, Ryu YW, Seo JH (2000) Production of xylitol in cell recycle fermentation of Candida tropicalis. Biotechnol Lett 22, 1625-8.

39. Kurtzman CP, Dien BS (1998) Candida arabinofermentas, a new L-arabinose fermenting yeast. Antonie 
Leeuwenhoek 74, 237-43.

40. Sambrook J, Russell DW (2001) Molecular Cloning: A Laboratory Manual, 3rd edn, Cold Spring Harbor Laboratory, Cold Spring Harbor, NY.

41. Kurtzman CP, Robnett CJ, Basehoar-Powers E (2008) Phylogenetic relationships among species of Pichia, Issatchenkia and Williopsis determined from multigene phylogenetic analysis, and the proposal of Barnettozyma gen. nov., Lindnera gen. nov. and Wickerhamomyces gen. nov. FEMS Yeast Res 8, 939-54.

42. Margaritis A, Bajpai P (1982) Direct fermentation of dXylose to ethanol by Kluyveromyces marxianus strains. Appl Environ Microbiol 44, 1039-41.

43. Hou X (2012) Anaerobic xylose fermentation by Spathaspora passalidarum. Appl Microbiol Biotechnol 94, 205-14.

44. El-Baz AF, Shetaia YM, Elkhouli RR (2011) Xylitol production by Candida tropicalis under different statistically optimized growth conditions. Afr J Biotechnol 10, 15353-63.

45. Furlan SA, de Castro HF (2001) Xylitol production by Candida parapsilosis under fed-batch culture. Braz Arch Biol Tech 44,125-8.

46. Praksham RS, Sreenivas Rao R, Hobbs PJ (2009) Current trends in biotechnological production of xylitol and future prospects. Curr Trends Biotechnol Pharm 3, 8-36.

47. Silva CJSM, Mussatto SI, Roberto IC (2006) Study of xylitol production by Candida guilliermondii on a bench bioreactor. J Food Eng 75, 115-9.

48. Sirisansaneeyakul S, Staniszewski M, Rizzi M (1995) Screening of yeasts for production of xylitol from Dxylose. J Ferment Bioeng 80, 565-70.

49. Guo C, Zhao C, He P, Lu D, Shen A, Jiang N (2006) Screening and characterization of yeasts for xylitol production. J Appl Microbiol 101, 1096-104.

50. Sreenivas Rao RS, Bhadra B, Shivaji S (2007) Isolation and characterization of xylitol-producing yeasts from the gut of colleopteran insects. Curr Microbiol 55, 441-6.

51. Ho NWY, Lin FP, Huang S, Andrews PC, Tsao TC (1990) Purification, characterization, and amino terminal sequence of xylose reductase from Candida shehatae. Enzym Microb Tech 12, 33-9.

52. Jeon YJ, Shin HS,Roger PL (2011) Xylitol production from a mutant strain of Candida tropicalis. Lett Appl Microbiol 53, 106-13.

53. Kavanagh K, Klimacek M, Nidetzky B, Wilson DK (2003) Structure of xylose reductase bound to $\mathrm{NAD}^{+}$ and the basis for single and dual co-substrate specificity in family 2 aldo-keto reductase. Biochem J 15, 319-26.

54. Lee JK, Koo BS, Kim SY (2003) Cloning and characterization of xyl1 gene, encoding an NADH-Preferring xylose reductase from Candida parapsilosis, and its function expression in Candida tropicalis. Appl Environ Microbiol 69, 6179-88. 